\title{
On Acquiring Expertise in Medicine
}

\author{
Henk G. Schmidt ${ }^{1}$ and Henny P. A. Boshuizen ${ }^{1}$
}

This article presents a new theory of expertise development in medicine and the empirical evidence available. This theory describes expertise development as the progression through a series of consecutive phases, each of which is characterized by functionally different knowledge structures underlying performance. The first phase is characterized by the accumulation of causal knowledge about disease and its consequences. Through experience with real cases, this knowledge transforms into narrative structures called illness scripts. The cognitive mechanisms responsible for this transition are: Encapsulation of elaborated knowledge into high level but simplified causal models or even diagnostic categories and tuning through the inclusion of contextual information. The third phase is characterized by the use of episodic memories of actual patients in the diagnosis of new cases. It is assumed that knowledge acquired in different phases form layers in memory through a sedimentation process. These knowledge sediments, although usually not applied any more in subsequent phases in the development of expertise, remain available for use when ontologically more recently acquired structures fail to produce an adequate representation of a clinical problem.

KEY WORDS: expertise development; encapsulation; contextual knowledge; illness scripts.

\section{INTRODUCTION}

In 1988, The Nature of Expertise edited by Chi, Glaser, and Farr was published. In the introduction, Glaser and Chi summarized the main findings of 40 years of research in this area of cognition. Their list consisted mainly of empirical generalizations, such as: experts excel mainly in their

${ }^{1}$ University of Limburg, The Netherlands. 
own domain; experts are faster than novices at performing the skills of their domain, and they quickly solve problems with little error; experts have superior short-term and long-term memory; experts see and represent a problem in their domain at a deeper (more principled) level than do novices; novices tend to represent a problem at a superficial level; and so forth. From their summary it seems as if only limited theoretical progress has been made since De Groot's seminal work on expertise in chess published in 1946.

There may be several reasons for this disappointing observation, three of which are offered here. First, most research into expertise in various domains has been largely descriptive. Investigators, following De Groot's (1946) early example, have been mainly interested in demonstrating differences in performance between subjects of different levels of expertise without considering the mechanisms underlying these performance differences. In order to make progress in an area, studies are needed that try to explain the empirical phenomena described. Second, the preferred method in this area is quasi-experimental comparison of performance of subjects known to be of different expertise. Although informative, this approach is a relatively weak one, because it does not allow for active manipulation of variables of interest. Third, unlike other areas in cognitive research (e.g., memory), the field appears to lack stable empirical phenomena whose explanation may contribute to a better understanding of the nature of expertise and of how it develops in human beings.

In this article, we hope to demonstrate that a more explanationoriented, experimental approach may open new avenues to understanding expertise and its development. This approach tests specific hypotheses about the nature of the cognitive structures responsible for expert performance and concentrates on apparent anomalies reported in the literature.

The domain of interest, medical expertise, is an appealing subject for study. Like other domains, such as solving physics problems (Chi, Feltovich, and Glaser, 1981), reconstructing historical events (Whineburg, 1991), or understanding social science problems (Voss, Greene, Post, and Penner, 1983), the problems of medicine are ill-defined and take many years to achieve an acceptable level of mastery. In addition, the practice of medicine involves diagnostic reasoning based on underlying principles or rules used to arrive at a solution. Like medicine, many problems in the real world require some kind of diagnosis based on conceptual knowledge to characterize or understand a situation and act upon it in an appropriate way.

In the next sections, first our theory on the development of medical expertise will be presented. Next, empirical evidence derived from our own research project and from other researchers will be discussed as well as 
unanswered questions that need further investigation. We will conclude by a discussion of our research plans and of possible educational implications of the theory and the data available.

\section{A MODEL OF EXPERTISE DEVELOPMENT}

Current theories dealing with expertise (e.g., Chi, Feltovich, and Glaser, 1981; Chi, Glaser, and Rees, 1982; Lesgold, Rubinson, Feltovich, Glaser, Klopfer, and Wang, 1988) consider its development largely a process of extension of causal knowledge about a domain. Through education and experience, subjects acquire more relevant concepts and develop richer and more meaningful relations between them leading to a better structure for the tasks at hand. Lesgold et al. (1988), for instance, assume that expert radiologists' superior diagnostic skills are based on a richer biomedical knowledge base and on a deeper understanding of the causes and consequences of disorders observed on X-rays than intermediates and novices in that area have available. For these theorists, the development of expertise seems to be essentially a linear process, mainly characterized by the acquisition of more, and better structured, knowledge. Vosniadou and Brewer (1987), following Carey (1985), describe this position as the "weak restructuring" postulate, as opposed to a "radical" restructuring view of expertise development. In the latter view, novices do not just have a poorer knowledge base than experts, but their knowledge is different; different in terms of its concepts, different also in structure. From the radical restructuring perspective, the development of expertise would involve, in addition to a process of knowledge elaboration, several shifts in the knowledge base resulting in new conceptualizations of the domain.

The theory proposed in this article takes this radical view on expertise development. It postulates that the development of expertise in medicine can only be properly understood by assuming certain kinds of knowledgebase shifts in the course of growth towards expertise, resulting in a developmental process that can be characterized by a sequence of distinct phases in which the learner uses qualitatively different knowledge bases while diagnosing a case.

According to this central postulate, experienced physicians, while diagnosing routine cases, are operating upon knowledge structures that are distinctly different from those of novice physicians and students. These expert knowledge structures, which we describe as "illness scripts," emerge from continuing exposure to patients and are, therefore, largely the result of extended practice. These illness scripts contain relatively little knowledge 
about pathophysiological causes of symptoms and complaints but have a wealth of clinically relevant information about disease, its consequences, and the context under which the illness develops. Illness scripts seem to exist at various levels of generality, ranging from representations of disease categories to representations of individual patients seen before. Indeed, one salient feature of our theory is the notion that physicians actually use the memories of previous patients while diagnosing a new case (cf. Allen, Norman, and Brooks, 1988). Our position as to how these expert structures develop can be summarized as follows.

In the course of their medical training, students acquire rich, elaborated causal networks explaining the causes and consequences of disease in terms of general underlying biological or pathophysiological processes. However, through extensive and repeated application of knowledge acquired and, particularly, through exposure to patient problems, these declarative networks become encapsulated ${ }^{2}$ into diagnostic labels or high level, simplified causal models explaining signs and symptoms. In this encapsulation process, low-level, detailed concepts are clustered together, resulting in higher-level concepts. These higher-level concepts replace the elaborate causal networks in expert clinical reasoning. At the same time, a transition takes place from a network-type of knowledge organization to the narrative structures we refer to as "illness scripts." While solving a problem, a physician searches for an appropriate script. When he has selected one (or a few), he will tend to match its elements to the information provided by the patient. In the course of this script-verification process, the script becomes instantiated. Instantiated scripts, in turn, do not become decontextualized after use but remain available in memory as episodic traces of previously diagnosed patients and will be used in the diagnosis of future similar problems. Finally, knowledge structures acquired during the different phases of development, pathophysiological networks, encapsulated structures, illness scripts, and episodic traces of instantiated scripts, do not decay; neither do they become inert, nor inaccessible. They sedimentate into multiple "layers" which are accessed when ontogenetically more recently acquired structures fail in producing an adequate representation of a clinical problem (Schmidt, Norman, and Boshuizen, 1990).

\footnotetext{
${ }^{2}$ The notion of knowledge encapsulation is analogous to the notions of knowledge compilation (Anderson, 1983, 1987), and chunking (Laird, Rosenbloom, and Newell, 1986), all leading to a marked acceleration of search through a knowledge base. The term "encapsulation" was, however, introduced to emphasize the abbreviations in search processes resulting from knowledge application without rendering the original knowledge base inaccessible. See for details Schmidt and Bashuizen (1992).
} 


\section{EVIDENCE FOR THE ACQUISITION OF ELABORATE CAUSAL NETWORKS AND THEIR SUBSEQUENT ENCAPSULATION}

\section{Intermediate Effect Studies}

Evidence supporting the idea of transitory phases in expertise development is partly based on studies of the way subjects of different levels of medical expertise represent clinical cases describing the complaints, signs, and symptoms of a particular patient. A typical experiment consists of four elements: (a) presentation of a clinical case, (b) recall of the case, (c) explanation of the signs and symptoms in terms of underlying pathophysiology, and (d) diagnosis. Researchers working in this paradigm (cf. Patel and Groen, 1986) assume that the nature and the amount of the recall are dependent on the knowledge structures activated to comprehend the text; hence the recall of the case depicts the subject's problem representation or "problem model" (Kintsch and Greeno, 1985), while the pathophysiology protocol is assumed to directly reflect the activated knowledge structures used in the process of comprehending the case.

Studies of clinical case processing by students in the early years of medical education almost invariably show a steep increase in the number of propositions recalled. Boshuizen (1989) for instance, presented $48 \mathrm{sec}-$ ond-, fourth- and fifth-year students ${ }^{3}$ and 16 family physicians with either a case of prostatitis or pancreatitis. Subsequently, the subjects were required to recall whatever they could remember from the case. She found that the fourth and fifth-year students recalled almost twice as many items from the case as the second-year students. These results suggest that the knowledge structures mobilized in the processing of clinical cases become more elaborate, richer, and better organized as a function of developing expertise. However, the family physicians involved in the Boshuizen study recalled significantly less information from the case than the fourth- and fifth-year students. This finding is in accordance with other recall studies (e.g., Claessen and Boshuizen, 1985; Hassebrock, Bullemer, and Johnson, 1988; Muzzin, Norman, Feightner, and Tugwell, 1983; Patel and MedleyMark, 1986). These studies also demonstrate that recall performance of subjects involved in patient care actually decreases with increasing expertise. Expert physicians typically recall fewer propositions than do intermediatelevel medical students who recall more than novices. This phenomenon has become known as the "intermediate effect" in clinical case representation studies.

${ }^{3}$ Medical students in the Netherlands follow a 6-year curriculum. 
In an attempt to find the source of the intermediate effect, Schmidt, Boshuizen, and Hobus (1988) asked subjects of five different levels of expertise, ranging from lay persons (first-year health sciences students) to internists, to study a case of acute bacterial endocarditis. The number of propositions recalled reflected a clear-cut intermediate effect, fourth-year medical students having the highest recall. In addition, Schmidt et al. asked subjects to explain the signs and symptoms displayed in the case description in terms of underlying pathophysiology in an attempt to capture the knowledge active during processing of the endocarditis case. These pathophysiology protocols were segmented into propositions. The data showed a strong intermediate effect in the number of propositions produced in the pathophysiology protocols as well. Recall and pathophysiology output were correlated .57. Qualitative analyses of the pathophysiology networks, derived from the subjects' protocols, showed that extensive knowledge encapsulation takes place as soon as subjects become exposed to actual patients, encapsulation being strongest among the internists. For instance, fourth year preclinical students often try to account for almost every cue embedded in the text of the endocarditis case, using whatever biological or pathophysiological knowledge is available, suggesting that extensive processing has taken place. Explanation is at a detailed level; quite a few of the propositions produced include basic physiological mechanisms. By contrast, the internists' propositions include only a few cues provided by the case, and explanations are highly encapsulated. There is no reference to causative agents like bacteria nor to pathophysiological processes such as inflammation reaction, seeding of bacteria into the bloodstream, and lodging on the aortic valve, although all these concepts are important in the explanation of acute bacterial endocarditis. These concepts seem to be embedded in a few highly condensed - often diagnostic - concepts (e.g., sepsis, aorta insufficiency, embolisms) and their relationships. Explanatory concepts at the (patho)physiological level have become encapsulated into concepts of greater generality.

In the same experiment, Schmidt et al. (1988) manipulated the amount of time available for processing the clinical case. They were able to show that if processing time is restricted, the intermediate effect in both recall and pathophysiology disappears. Instead, a weak positive linear relation with expertise level is found. From these data, they conclude that students and experienced physicians seem to represent clinical cases in different ways, applying functionally different knowledge. Provided they have sufficient time, medical students consciously process causal pathophysiological knowledge activated by cues embedded in the text. Physicians, however, only pick up information that is directly relevant to the solution of the problem without much pathophysiological reasoning, simply because they possess readymade cognitive structures, against which relevant information is matched. 
A shortcoming of the 1988 study was that a causal hypothesis concerning the effect of pathophysiology activation on case processing was tested with an indirect method. The amount of pathophysiology activation was inferred from the explanations provided only after the actual processing had taken place. Therefore, a second experiment was conducted in which subjects' prior knowledge of pathophysiology was primed ${ }^{4}$ by having them recall whatever they knew about endocarditis for either $30 \mathrm{sec}$ or $3.5 \mathrm{~min}$. Subsequently, both groups studied the endocarditis case for $30 \mathrm{sec}$ (Schmidt and Boshuizen, 1993). It was hypothesized that students' recall, at the novice and intermediate levels, would be mediated by the amount of knowledge activated, while experts' recall would not. Hence, it was expected that differences in the amount of priming would only affect students' recall. Two effects of the manipulation of amount of pathophysiology activation were found. The first was that students who had only $30 \mathrm{sec}$ to activate relevant prior knowledge remembered less information from the case than did those who had $3 \mathrm{~min}$ and $30 \mathrm{sec}$ for activation, although both groups had the same amount of time available to study the case. The internists' recall turned out to be entirely independent of the experimental manipulation. Second, an intermediate effect was found in the data. Students of intermediate levels recalled more information from the case than did novices and experts. (See Figure 1 on the next page.) These findings further support the notion that the intermediate effect in clinical case recall indeed results from the activation of elaborate, causal, pathophysiological knowledge by students of intermediate levels of expertise. In addition, it demonstrates that experienced physicians process clinical information using knowledge structures distinctively different from those of students.

\section{Think-Aloud Studies}

A second line of research illustrating the relatively scarce application of biomedical concepts by expert physicians involves naturalistic observations and think-aloud studies. Boshuizen, Schmidt, and Coughlin (1987), for instance, using a think-aloud methodology, showed that general practitioners rarely refer to pathophysiological concepts while reasoning about a case, whereas students use pathophysiological concepts extensively in the same situation (see also the review by Patel, Evans, and Groen, 1989).

\footnotetext{
${ }^{4}$ This technique was adapted from Peeck, Van den Bosch, and Kreupeling (1982) who demonstrated that priming of prior knowledge previous to the processing of a relevant text facilitates comprehension and recall of that text.
} 


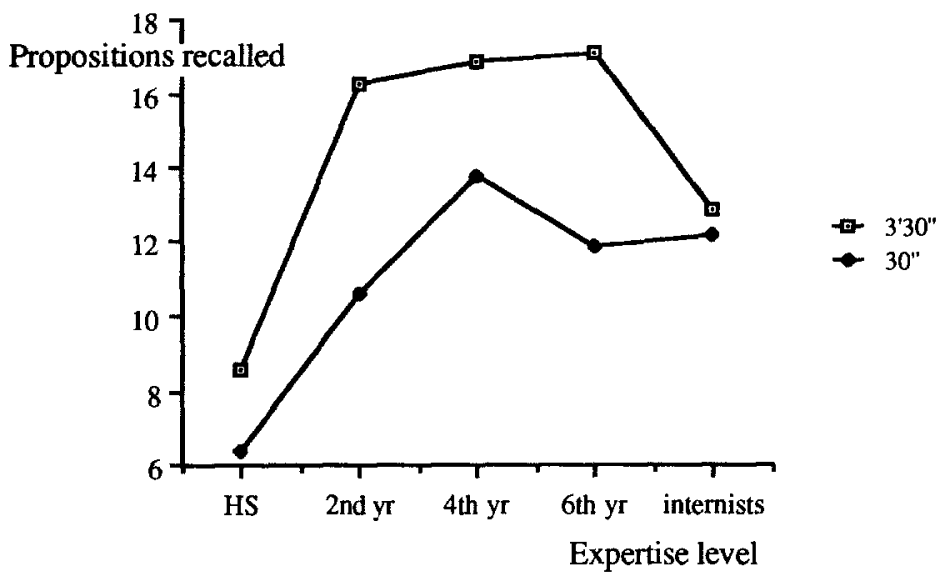

Fig. 1. Number of propositions recalled of a clinical case studied for $30 \mathrm{sec}$ by subjects of five different levels of expertise ranging from lay persons to experts, after priming of relevant knowledge during $3 \mathrm{~min}$ and $30 \mathrm{sec}$ or during only 30 sec. (Schmidt and Boshuizen, 1993).

Boshuizen and Schmidt (1992) hypothesized that three mutually exclusive cognitive processes may account for this phenomenon: (a) After a certain phase in the development toward expertise, biomedical knowledge becomes rudimentary: Large parts of the expert's biomedical knowledge (especially detailed knowledge) are no longer retrievable. (b) Biomedical knowledge becomes inert: It is still available to medical experts, and it can be activated when directly addressed, but it is not used in diagnostic reasoning; clinical knowledge is applied instead. (c) In the course of the years, biomedical knowledge becomes encapsulated and is integrated into clinical knowledge (i.e., in clinical reasoning, experts apply encapsulated knowledge, which is directly linked to associated, detailed, deep-level knowledge and which can be retrieved whenever necessary). Using a combined on-line and post hoc methodology, Boshuizen and Schmidt tested these hypotheses. Twenty subjects of different levels of expertise were presented with a set of 20 cards containing the signs and symptoms of a patient suffering from acute pancreatitis. The subjects were requested to think aloud while processing this information. After finishing this task, they were asked to provide a diagnosis and to explain the case in terms of underlying pathophysiological processes. The think-aloud and pathophysiology protocols of each subject were parsed into propositions and subsequently compared. The idea was that if biomedical knowledge would have become rudimentary and no longer retrievable, one would find a decrease of this category of knowledge 
with increasing levels of expertise in both the think-aloud and the pathophysiology protocols. If, however, the knowledge were still available but not used any more (at least not in rather straightforward cases such as this pancreatitis case), one might expect a decrease in references to biomedical concepts in think-aloud protocols associated with an increase in expertise, but an increase in the protocols collected afterward. This would indicate that the knowledge was still there, but that it had become inert and was not activated any more while solving problems. Finally, if biomedical concepts would have become encapsulated, one would expect a decrease in the think-aloud protocols but, at the same time, an increasing linkage between concepts used in these protocols and the post hoc explanations. The data corroborated the encapsulation-hypothesis. Students produced generally more biomedical propositions while thinking aloud, but the knowledge activated was less intimately linked to the knowledge represented in the post hoc explanations. Among the novices, the amount of overlap was only $14.9 \%$. This percentage increased to $56.2 \%$ in the expert group. The linear component associated with this trend was highly significant. Boshuizen and Schmidt (1992) concluded that experts use biomedical knowledge in a tacit way, because in the course of becoming an expert this type of causal knowledge becomes encapsulated into clinical concepts.

In summary, quantitative and qualitative studies concentrating on the intermediate effect in clinical case recall and studies of the use of biomedical concepts in clinical reasoning both seem to indicate that preclinical students primarily construct problem representations using detailed biomedical knowledge. In order to make sense of a case, they have to activate and process this knowledge in a conscious fashion, a cognitive activity that takes considerable time because no ready-made structures are available. As soon as patients are encountered, a shift emerges. The confrontation with similar patients for a couple of times (on a cardiology ward, for instance) induces "short cuts" in the student's reasoning. After a while, the student does not need extensive pathophysiological processing any more; the use of a smaller number of more inclusive concepts suffices for understanding. Encapsulation begins to take place. The student begins to construct illness scripts for understanding.

\section{EVIDENCE FOR THE EXISTENCE OF ILLNESS SCRIPTS AS COGNITIVE ENTITIES IN EXPERTS}

According to Feltovich and Barrows (1984), students and physicians, while seeing patients, pick up information about the circumstances under which specific diseases are likely to occur. Examples of these circumstances 
Table I. Generic Illness Script ${ }^{a}$

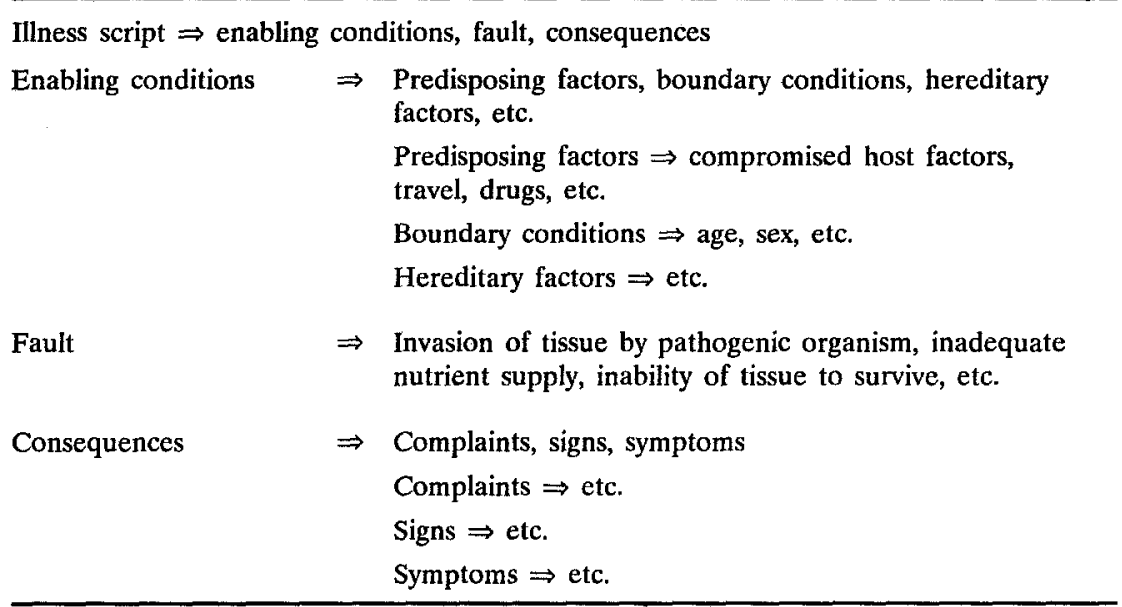

${ }^{a}$ From Feltovich and Barrows (1984).

are life style, working environment, previous disease history, age, and sex. These circumstances are not necessarily causally related to certain diseases, but enable them. They more or less "predispose" people to get a disease. Somebody does not get a cardiac infarction as a result of being a male and older than 40 , but people of that sex and age more often are victims of a cardiac infarction than are other people. Hence, knowledge of these factors helps in diagnosing that illness. So, gradually, physicians develop in their minds typical patient histories related to certain sets of symptoms and a certain diagnosis, called "illness scripts." Illness scripts thus contain the physician's encapsulated pathophysiological knowledge of the disease and its consequences, in addition to clinical knowledge of the constraints under which a disease occurs. They are narrative structures, containing prototypical information about a disease, which, when activated, guide a clinician through a case and support him in looking for relevant cues. Table I shows the Feltovich and Barrows generic model of an illness script.

An implication of narrative structures is that they tend to be recalled in a fixed order, and this is precisely what has been demonstrated in various studies. Claessen and Boshuizen (1985) for instance, found that, with increasing expertise, subjects increasingly tend to group elements together in a way compatible with the illness script format. Their study was flawed, however, in the sense that the stimulus materials already had a script structure. Coughlin (1986), however, presented novices and experts with either 
a scripted or a scrambled version of a clinical case. Whereas the recall of the novices was greatly affected by the experimental manipulation, the recall of the experts displayed the typical script-like structure, irrespective of the structure of the text presented. These data indicate that, while processing a case, experts represent patient information in pre-existing linear structures that have a fixed order.

In a study of the role played by enabling conditions in the activation of an appropriate script, Hobus, Schmidt, Boshuizen, and Patel (1987) presented experienced and inexperienced family physicians with 32 clinical cases, each consisting of a patient chart, a picture of the patient, and his main complaint. The patient chart and the picture displayed enabling-conditions information such as age, sex, previous diseases, occupation, drug use, and so forth. The information was presented on slides and could only be studied for a limited amount of time. After each case, the physician was asked to state a preliminary diagnostic hypothesis. Afterward the subjects were asked to recall as much as they could about the patients presented. The complaint associated with each case, together with the subject's own diagnosis of that case, were used as a cue. The results showed that experienced physicians accurately diagnosed almost $40 \%$ of the cases, whereas the novice physicians were accurate in $27 \%$. In addition, the experienced physicians recalled significantly more contextual information about the patients presented. This finding led Hobus and colleagues to believe that experts' unequaled diagnostic performance was based on their superior ability to interpret and use the enabling-conditions information provided. This assumption was tested in a second experiment by Hobus, Hofstra, Boshuizen, and Schmidt (1988). In this experiment, the experimental manipulation consisted of presenting the complaint either with or without the patient chart and the picture. The task of the subjects was to formulate a preliminary diagnostic hypothesis based on the available information. When presented with all the information, the experienced physicians produced twice as many accurate diagnoses as compared with the inexperienced physicians. These results corroborated the findings of the 1987 study. However, the number of accurate diagnoses produced by the experienced physicians decreased to almost $50 \%$ when no patient chart and picture were provided, whereas the inexperienced physicians were only marginally affected. These results clearly demonstrate the overriding importance of enabling conditions in the activation of relevant illness scripts. In addition, they demonstrate that this knowledge is only acquired through extended practice. It is the daily confrontation with patient problems above all else that provides the opportunities for acquiring contextual knowledge essential to the development of expertise. 


\section{EVIDENCE FOR THE USE OF INSTANCES IN THE DIAGNOSIS OF NEW CASES}

According to Brooks (1987), the human ability to categorize objects is as much determined by earlier experience with an object that resembles the present one as it is determined by abstract, decontextualized rules linking sets of features to category names. His work prompted Norman and his associates (Allen, Norman, and Brooks, 1988; Norman, Rosenthal, Brooks, Allen, and Muzzin, 1989) to test the hypothesis that diagnostic performance of physicians and medical students is influenced by exposure to previous patients who looked like the present patient. In one experiment (Allen et al., 1988), first-year medical students were required to study a set of rules to diagnose dermatological diseases. Next, they were presented with 24 slides displaying six different diseases to practice the rules. The slides were shown several times, and feedback was provided after each attempt in order to ensure the learning of the rules. The experimental manipulation involved the choice of the slides used in the practice set. Two different sets were used, with the intention of biasing the diagnosis of slides in the test series. For example, if one of the ambiguous test slides was a case of lichen planus on the wrist which might be confused with contact dermatitis, then one practice set would contain a similar lichen planus on the wrist, and the other would contain a similar appearing contact dermatitis on the wrist. All subjects received the same test slides. If subjects' diagnostic behavior was based on the application of the rules, no incorrect diagnoses were to be expected, because the location of the skin disease or other contextual aspects were not in any way part of the rules (in fact, they were irrelevant to the diagnosis of the particular disease), and subjects had the rules in front of them. If, however, their behavior was influenced by seeing similar exemplars from another disease, incorrect diagnoses were to be expected. When shown an appropriate analogy, subjects correctly diagnosed the test slide $94 \%$ of the time. Conversely, when shown a look-alike but wrong analogy, only $38 \%$ of subjects selected the correct diagnosis. The influence of prior examples was apparent even after a week and exposure to further examples. These data suggest that diagnostic performance is at least to the same extent determined by previous experience with similar exemplars as it is by the application of diagnostic rules. Studies undertaken with experts show similar results. In conclusion, the available evidence seems to suggest that physicians store experiences with concrete patients in memory and use these episodic traces in the diagnosing of similar new patients. 


\section{EVIDENCE FOR THE AVAILABILITY OF MULTIPLE-LAYERED KNOWLEDGE STRUCTURES IN EXPERTS}

In a series of studies, Patel and her colleagues (e.g., Patel, Evans, and Chawla, 1986b) have demonstrated that the amount of pathophysiological reasoning in protocols of subjects decreases with an increase in level of expertise. Thus, the more expert one is, the less one seems to rely on causal explanations to arrive at a diagnosis. These findings are in accordance with the studies cited above; they imply that the nature of the cognitive structures upon which subjects operate changes with level of expertise. In addition, however, Patel was able to show the same phenomenon to occur among physicians of the same level of expertise. In a study by Patel, Arocha and Groen (1986a), experts from two distinct medical specialties were compared on tasks both within and across their domains of expertise. They demonstrated that physicians solving a problem outside their domain of expertise produced more pathophysiological explanations than within their domain of expertise. In addition, within the same domain, experts who were more involved in clinical research and, hence, saw fewer patients also tended to rely more on pathophysiological explanation than did their colleagues who saw patients on a daily basis. Finally, Boshuizen (1989) demonstrated that experts are able to retrieve pathophysiological knowledge, dependent on the specific task one requires from them, which means that this kind of knowledge does not decay or become inaccessible. Such knowledge is just not used in detail when one is confronted with routine cases, but it is available when a difficult case is presented and when available illness script knowledge does not apply or is not available. These studies seem to support the notion that experts have knowledge available in different layers. To experts, illness-script knowledge represents the preferred level. However, when a case is presented for which no knowledge of that kind exists they will "regress" to sub-expert or advanced-student levels of reasoning, involving the application of causal, pathophysiological reasoning.

\section{SUMMARY AND PERSPECTIVE}

The theory of expertise development in medicine presented in this paper deviates from other proposals (e.g., Lesgold, 1984) in several ways. The first is that it assumes that the development of expertise can be described as the progression through a series of transitory phases. Second, knowledge acquired during different phases of expertise development has 
a distinctly different organization, earlier forms tending to be organized in causal networks, more recent forms being structured as scripts. Third, it is assumed that knowledge acquired in different phases form layers in memory through a sedimentation process. Fourth, these knowledge sediments, although usually not applied any more in subsequent phases in the development of expertise, remain available for use when more recently acquired structures fail in producing an adequate representation of a clinical problem. Fifth, episodic traces of clinical problems previously analyzed seem to be extensively used in the representation and solution of new cases.

This theoretical framework establishes an agenda for future research. One aspect to be pursued is knowledge encapsulation, especially the question whether encapsulation is a gradual or a one-trial learning process. Another research question concerns changes in the structure and function of illness scripts during development toward expertise. The illness-script studies described in this article only cover the more advanced stretch of the developmental path (viz. the period after graduation from medical school). Earlier stages also have to be investigated. Furthermore, the process of activation and verification of hypotheses will have to be studied in more detail. A final research line addresses the flexibility in expert reasoning. The questions of whether, when, and how experts revert to ontogenetically less recent knowledge structures when trying to diagnose or treat a patient must especially be investigated.

The theory and the associated empirical data available suggest that several aspects of the learning process of medical students might be enhanced by educational measures. During the first phase of knowledge acquisition, integration of knowledge from different subject matters should be stimulated in order to acquire valid, integrated, and elaborate causal networks. A curriculum consisting of a set of parallel and sequenced courses without moments of integration does not help the student to reach this goal. Furthermore, our research shows the necessity of illness scripts in clinical reasoning. Educators can guide students' knowledge encapsulation and illness-script formation by providing representative cases of representative diseases from a specific category. Now, especially during internships, students' attention is too often drawn toward rare diseases and strange cases of ordinary diseases, which they must observe in order to take advantage of such a rare opportunity. Instead, their attention should be drawn primarily toward the more ordinary patients. Finally, students might be sensitized to the importance of enabling-conditions information in hypothesis generation and verification. This aspect, which seems to be characteristic for expert clinical reasoning, is lacking in sub-experts. Informal observations suggest that students deny the importance of enabling- 
conditions information. Apparently, they are of the opinion that clinical reasoning must be based on "solid" information such as signs and symptoms. This analysis suggests that a curriculum that supposes practical experience will suffice for the development of relevant, flexible illness scripts relies too heavily on the epidemiology at one specific place, during one specific, possibly short time of the year (e.g., a very specialized hospital ward, in winter). Instead, it is required that students are presented with relevant, representative problems and cases, in order to steer their learning processes.

\section{ACKNOWLEDGMENTS}

Parts of this article were presented at the Annual Meeting of the American Educational Research Association, Boston, Massachusetts, April 1990. Completion of this article was supported by a grant from the Spencer Foundation to the second author.

\section{REFERENCES}

Allen, S. W., Norman, G. R., and Brooks, L. R. (1988). Effects of Prior Examples on Rule-Based Diagnostic Performance. Paper presented at the Annual Meeting of the American Educational Research Association, New Orleans.

Anderson, J. R. (1983). The Architecture of Cognition, Harvard University Press, Cambridge, MA. Anderson. J. R. (1987). Skill acquisition: Compilation of weak-method problem solutions. Psychol. Rev. 4: 92-210.

Boshuizen, H. P. A. (1989). De Ontwikkeling van Medische Expertise; een CognitiefPsychologische Benadering. (On the Development of Medical Expertise; A Cognitive Psychological Approach.) Doctoral dissertation, University of Limburg, Thesis Publishers, Haarlem, The Netherlands.

Boshuizen, H. P. A., and Schmidt, H. G. (1992). The role of biomedical knowledge in clinical reasoning by experts, intermediates and novices. Cognit. Sci. 16: 153-184.

Boshuizen, H. P. A., Schmidt, H. G., and Coughlin, L. D. (1987). On-Line Representation of a Clinical Case and the Development of Expertise. Paper presented at the Annual Meeting of the American Educational Research Association, Washington, D.C.

Brooks, L. R. (1987). Decentralized control of categorization: The role of prior processing episodes. In Neisser, U. (ed.), Concepts and Conceptual Development, Cambridge University Press, New York.

Carey, S. (1985). Conceptual change in childhood. MIT Press, Cambridge, MA.

Chi, M. T. H., Feltovich, P. J., and Glaser, R. (1981). Categorization and representation of physics problems by experts and novices. Cognit. Sci. 5: 121-152.

Chi, M. T. H., Glaser, R., and Rees, E. (1982). Expertise in problem solving. In Sternberg, R. (ed.), Advances in The Psychology of Human Intelligence, Erlbaum, Hillsdale, NJ, pp. 7-76.

Chi, M. T. H., Glaser, R., and Farr, M. J. (1988). The Nature of Expertise. Erlbaum, Hillsdale, NJ.

Claessen, H. F. A. and Boshuizen, H. P. A. (1985). Recall of medical information by students and doctors. Med. Educ. 19: 61-67. 
Coughlin, L. D. J., (1986). The Effect of Randomization on the Free Recall of Medical Information by Experts and Novices, M. A. Thesis, McGill University, Montréal, Canada.

DeGroot, A. D. (1946). Het Denken van den Schaker. (Thinking Processes in Chess Players.) Noord Holland, Den Haag, The Netherlands.

Feltovich, P. J. and Barrows, H. S. (1984). Issues of generality in medical problem solving. In Schmidt, H. G., and de Volder, M. L. (eds.), Tutorials in Problem-Based Learning, Van Gorcum, Assen.

Hassebrock, F., Bullemer, P., and Johnson, P. E. (1988). Wenn Less is More: Selective Memory of Problem-Solving Experts. Paper presented at the Annual Meeting of the American Educational Research Association, New Orleans.

Hobus, P. P. M., Schmidt, H. G., Boshuizen, H. P. A., and Patel, V. L. (1987). Contextual factors in the activation of first diagnostic hypotheses: Expert-novice differences. Med. Educ. 21: 471-476.

Hobus, P. P. M., Hofstra, M. L., Boshuizen, H. P. A., and Schmidt, H. G. (1988). De context van de klacht als diagnosticum. (The context of the complaint as a diagnostic tool.) Huisarts en Wetenschap 31: 261-267.

Kintsch, W., and Greeno, J. G. (1985). Understanding and solving word arithmetic problems. Psychol. Rev. 92: 109-129.

Laird, J. E., Rosenbloom, P. S., and Newell, A. (1986). Chunking in SOAR: The anatomy of a general learning mechanism. Machine Learn. 1: 11-46.

Lesgold, A. M. (1984). Acquiring expertise. In Anderson, J. R., and Kosslyn, S. M. (eds.), Tutorials in Learning and Memory, Freeman, San Francisco, CA.

Lesgold, A., Rubinson, H., Feltovich, P. J., Glaser, R., Klopfer, D., and Wang, Y. (1988). Expertise in a complex skill: Diagnosing X-ray pictures. In Chi, M. T. H., Glaser, R., and Farr, M. (eds.), The Nature of Expertise, Earlbaum, Hillsdale, NJ, pp. 311-342.

Muzzin, L. J., Norman, G. R., Feightner, J. W., and Tugwell, P. (1983). Expertise in Recall of Clinical Protocols in Two Specialty Areas. Proceedings of the 22nd Conference on Research in Medical Education, Washington, D.C.

Norman, G. R., Rosenthal, D., Brooks, L. R., Allen, S. W., and Muzzin, L. J. (1989). The development of expertise in dermatology. Arch. Dermatol. 125: 1063-1068.

Patel, V. L., and Groen, G. J. (1986). Knowledge-based solution strategies in medical reasoning. Cognit. Sci. 10: 91-116.

Patel, V. L., and Medley-Mark, V. (1986). Relationship Between Representation of Textual Information and Underlying Problem-Representation in Medicine. CME Report CME86-CS1, McGill University, Montreal.

Patel, V. L., Arocha, J. F., and Groen, G. J. (1986a). Strategy selection and degree of expertise in medical reasoning. In Proceedings of the 8th Annual Conference of the Cognitive Science Society, Lawrence Erlbaum, Hillsdale, NJ.

Patel, V. L., Evans, D. A., and Chawla, A. S. (1986b). Predictive versus diagnostic reasoning in the application of biomedical knowledge. In Proceedings of the 8th Annual Conference of the Cognitive Science Society, Lawrence Erlbaum, Hillsdale, NJ.

Patel, V. L., Evans, D. A., and Groen, G. J. (1989). Biomedical knowledge and clinical reasoning. In Patel, V. L., and Evans, D. A. (eds.), Cognitive Science in Medicine: Biomedical Modeling, MIT Press, Cambridge, MA., pp. 53-112.

Peeck, J., Van den Bosch, A. B., and Kreupeling, W. J. (1982). The effect of mobilizing prior knowledge on learning from text. J. Educ. Psychol. 74: 771-777.

Schmidt, H. G., and Boshuizen, H. P. A. (1992). Encapsulation of biomedical knowledge. In Evans, D. A., and Patel, V. L. (eds.), Advanced models of cognition for medical training and practice, Springer Verlag, New York, NY.

Schmidt, H. G., and Boshuizen, H. P. A. (1993). On the origin of intermediate effects in clinical case recall. Memory \& Cognition, in press.

Schmidt, H. G., Boshuizen, H. P. A., and Hobus; P. P. M. (1988). Transitory stages in the development of medical expertise: The "intermediate effect" in clinical case representation studies. In Proceedings of the Cognitive Science Society Meeting, Lawrence Erlbaum, Hillsdale, NJ. 
Schmidt, H. G., Norman, G, R., and Boshuizen, H. P. A. (1990). A cognitive perspective on medical expertise: Theory and implications. Acad. Med. 65: 611-621.

Vosniadou, S., and Brewer, W. F. (1987). Theories of knowledge restructuring in development. Rev. Educ. Res. 57: 51-67.

Voss, J. F., Greene, T. R., Post, T. A., and Penner, B. C (1983). Problem-solving skill in the social sciences. In Bower, G. H. (ed.), The Psychology of Learning and Motivation: Advances in Research and Theory (Vol. 17), Academic Press, New York, pp. 165-213.

Whineburg, S. S. (1991). Historical problem solving: A study of the cognitive processes used in the evaluation of documentary and pictorial evidence. J. Educ. Psychol. 83: 73-87. 